La fondation "Sécurité des patients Suisse» a été fondée en 2003 par les Offices fédéraux de la santé publique et des assurances sociales, I'Académie Suisse des Sciences Médicales ainsi que de nombreuses organisations professionnelles dont la FMH. La FMH soutient financièrement et intellectuellement depuis des années divers projets de la fondation: Error \& Risk Analysis (ERA), CIRRNET, la gestion systématique des incidents / second victim, Safety Hot Spots, le tri téléphonique dans les soins de base, la sécurité des patients dans les soins des malades psychiques et actuellement un projet de subvention des revues de Mortalité et de Morbidité (RMM).

La FMH est persuadée que l'approche proactive de la fondation basée sur son solide enracinement ainsi que les différents projets en résultant apportent une contribution essentielle à la sécurité des patients en Suisse. De ce fait, la FMH soutient la semaine d'action pour la sécurité des patients 2017 du 17 au 22 septembre 2017 placée sous le thème du "Speak Up - quand le silence est dangereux». Vous trouverez ici: http://www.patientensicherheit.ch/aw/fr/aktionswoche-2017.html de plus amples informations concernant la semaine d'action.

\title{
«Parler peut sauver la vie - Speak Up!»
}

\section{Olga Frank}

Dr, collaboratrice Sécurité des patients Suisse

Dans le domaine de la santé, la communication entre les collaborateurs et collaboratrices des établissements spécialisés est un aspect d'importance cruciale. Toutefois, des structures hiérarchiques dominantes ou pressenties peuvent rendre cette communication difficile. S'y ajoutent la gestion des dilemmes éthiques, des émotions, des peurs ainsi que la pression du temps. Evoquer ses doutes quant à la sécurité n'est certes pas chose facile. Lorsque des collaborateurs gardent le silence malgré leurs doutes concernant la sécurité, cela résulte souvent d'un processus de mise en balance complexe. Certains craignent la remise en question de relations, alors que d'autres ne veulent pas placer leur interlocuteur en position de faiblesse ou l'inquiéter. Des résultats d'études réalisées aux Etats-Unis illustrent ce problème: dans l'étude «Silence skills» de Maxfield et al. [1] qui date de 2005, plus de la moitié des professionnels interrogés avaient observé des erreurs, des violations de règles ou un comportement incompétent. Toutefois, seule une personne sur dix a exprimé ses doutes.

\section{Une mauvaise communication peut causer des fautes systématiques}

Une mauvaise communication au sein de l'équipe, avec d'autres services et groupes professionnels, un travail d'équipe qui ne l'est pas, des interruptions, des distractions et le manque de formation en équipe sont non seulement identifiés comme des sources d'erreurs potentielles, mais aussi reconnus en tant que causes importantes de fautes systémiques accompagnées d'un risque de dommages en résultant. Les acteurs du système de santé se doivent de rester conscients que des erreurs peuvent survenir et qu'ils ont le devoir d'y faire face, c'est-à-dire de mettre en œuvre des dispositions afin qu'un incident n'ait pas de conséquences dommageables ou, pour le moins, que ses effets soient

Dans le domaine de la santé, la communication entre les collaborateurs et collaboratrices des établissements spécialisés est un aspect d'importance cruciale.

atténués. Confronter ses collègues et réagir si la sécurité des patients est compromise dans une situation donnée est l'une des nombreuses manières de contribuer à rendre un établissement de santé plus sûr. Ainsi, Speak Up est un élément qui contribue à la sécurité au niveau des soins aux patients. Malgré le potentiel de Speak Up pour une meilleure sécurité des patients, il n'est pas toujours évident d'y faire appel dans le quotidien hospitalier. Par conséquent, le potentiel de la ressource Speak Up est encore loin d'être épuisé. Mais pourquoi les collaborateurs ont-ils tant de difficulté à attirer l'attention de leurs collègues sur une erreur potentielle? Cela s'explique moins par une incompétence personnelle dans la pratique de Speak Up que par notre perception de l'environnement social et de ses réactions. Les obstacles se situent à trois niveaux: 1. l'organisation et ses contextes, 2 . la manière dont les relations humaines au poste de travail sont entrete- 


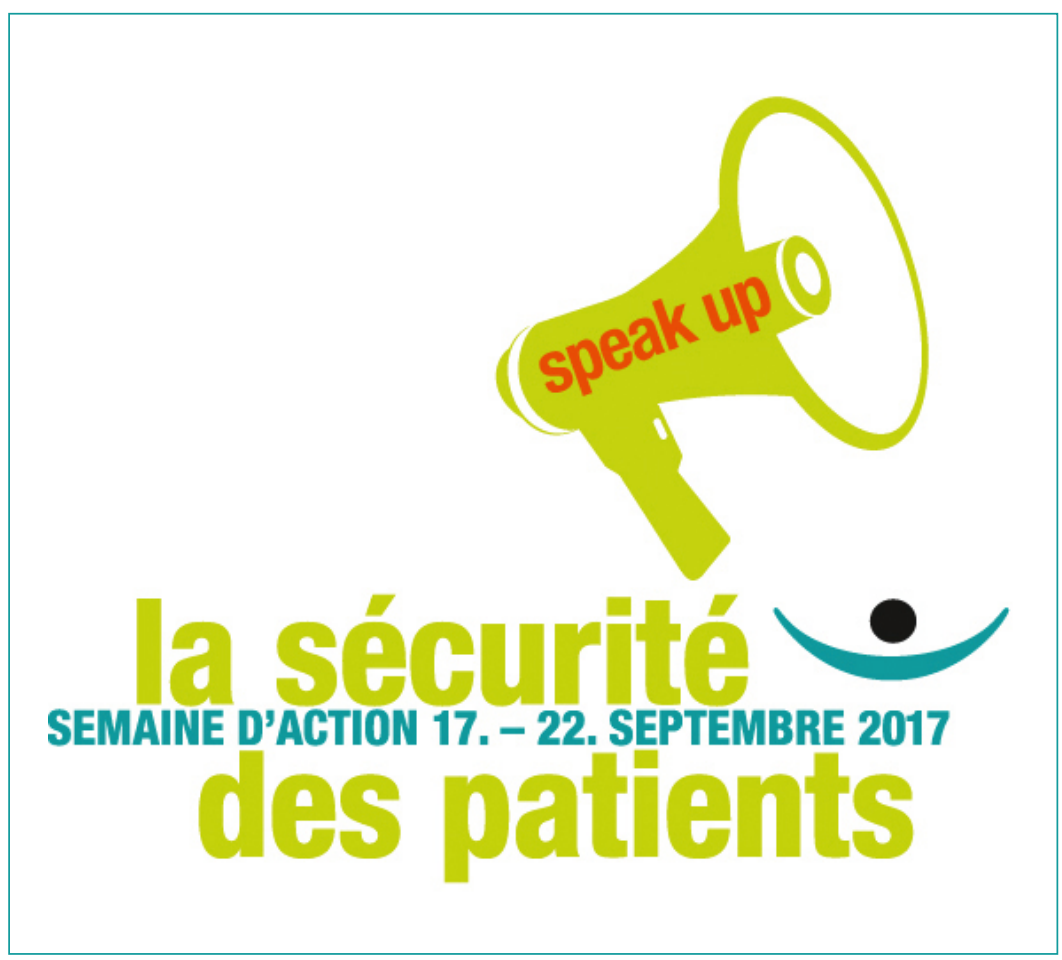

Correspondance: Dr Olga Frank Sécurité des patients Suisse Asylstrasse 77 CH-8032 Zurich Tél. 0432441484 frank[at] patientensicherheit.ch nues et perçues et 3. sa propre personnalité. Lors de l'établissement d'une culture Speak Up, il faut tenir compte de ces trois niveaux et mettre en place les préalables correspondants afin de faciliter l'évocation de doutes concernant la sécurité.

\section{Evoquer ses doutes quant à la sécurité n'est certes pas chose facile.}

Speak Up n'est pas une mesure indépendante des autres dispositions de sécurité, mais déploie souvent son effet dans l'interaction avec d'autres mesures. Il est donc plus facile d'y recourir, par exemple, si certaines règles ou certains standards existent et permettent de s'y référer. Speak Up - l'évocation des doutes concernant la sécurité, la soumission de propositions et l'expression d'idées - peut contribuer à rendre les soins aux patients plus sûrs. Il s'agit fondamentalement de protéger les patients des risques, d'éviter des erreurs aux collègues et d'apprendre ensemble à ne pas répéter les fautes.

Le projet consacré par la fondation Sécurité des patients Suisse à ce thème important a donné lieu à un cours et à une publication. En outre, la fondation concentrera sa semaine d'action 2017 pour la sécurité des patients sur ce sujet important. Elle invite tous les établissements de la santé à s'impliquer par des activités propres et à montrer tout ce qui est déjà intégré à leur organisation en matière de Speak Up.

Nous voulons donner un signal clair en faveur de Speak Up!

La semaine d'action Sécurité des patients 2017 aura lieu du 17 au 22 septembre. Cette semaine d'action vise à élargir encore le champ du thème Speak Up, à savoir aborder les doutes concernant la sécurité, tant auprès des établissements de santé que du grand public, et à en mettre l'importance en exergue. Nous voulons donner un signal clair en faveur de Speak Up! Tous les acteurs de la santé suisse seront intégrés à ce mouvement, raison pour laquelle un véritable engagement de chacun est souhaité. Le point décisif d'une telle action n'est pas son envergure, mais l'engagement dans le domaine de la sécurité des patients! Participez vous aussi à cette semaine d'action pour la sécurité des patients et, avec votre établissement, donnez un signe en faveur de Speak Up!

\section{Références et informations}

1 Maxfield D, Grenny J, McMillan R et al. Silence kills. The seven crucial conversations for healthcare. Aliso Viejo (CA): American Association of Critical Care Nurses; Association of perioperative Registered Nurses; VitalSmarts;2005.

2 Gehring K, Schwappach D. Speak Up. Quand le silence devient dangereux. Publication $n^{\circ} 8$ 2015. www.securitedespatients.ch

3 Formation continue Careum Cours journalier «Speak Up» https:// www.careum-weiterbildung.ch/angebot/kurse/detail. php?id=9034

4 Semaine d'action Sécurité des patients $2017 \mathrm{http}: / / \mathrm{www}$. semainedaction-securitedespatients.ch/aw/fr/aktionswoche-2017.html

\section{Crédit illustration}

Sécurité des patients Suisse 